\title{
UNIDADE PSIQUIÁTRICA EM HOSPITAL GERAL: CARACTERÍSTICAS DE ESTRUTURA E ORGANIZAÇÃO
}

\section{PSYCHIATRIC UNIT IN GENERAL HOSPITAL: CHARACTERISTICS OF STRUCTURE AND ORGANIZATION}

\section{UNIDAD PSIQUIÁTRICA EN EL HOSPITAL GENERAL: CARACTERÍSTICAS DE ESTRUCTURA Y ORGANIZACIÓN}

Tarcísia Castro Alves ${ }^{1}$, Margarita Antonia Villar Luis ${ }^{2}$.

\section{RESUMO}

Objetivo: caracterizar a unidade de psiquiatria do Hospital Geral, no tocante à estrutura, organização do trabalho, perfil profissional da enfermagem e tipo de paciente que recebe. Método: Trata-se de um estudo de caso de observação direta, abordagem qualitativa e quantitativa, com participação de dez profissionais de enfermagem. Resultados: Observou-se uma unidade com estrutura física moderna, pautada na intervenção multiprofissional. Profissionais de enfermagem jovens, do sexo feminino, com tempo de formação maior que 10 anos e mais de 3 anos de atuação na unidade psiquiátrica, mas cuja formação e experiência pouco se refere à saúde mental. Pacientes do sexo masculino, jovens, solteiros, em primeira internação, com longa taxa de permanência, sendo internados por situação de risco elevado de suicídio, seguidos de manifestações dos transtornos mentais relacionados ao uso de álcool e outras drogas. Conclusão: A unidade psiquiátrica, no hospital geral, possibilita o acesso ao cuidado de enfermagem e dos demais profissionais para uma atenção integral às necessidades das pessoas com transtorno mental. Contudo, há necessidade de maior formação da equipe de enfermagem para lidar, em saúde mental, nessas unidades, que se esbarram na reduzida oferta de cursos de especialização em algumas regiões do Brasil.

Descritores: Saúde mental; Enfermagem; Hospitais Gerais; Unidades de Internação.

\section{ABSTRACT}

Objective: to characterize the General Hospital's psychiatric unit according to its structure, work organization, nursing professional profile and the type of patient it receives. Method: This is a direct observation case study of qualitative and quantitative nature, with the participation of ten nursing professionals. Results: A unit with a modern physical structure based on multiprofessional intervention was identified. Young female nursing professionals with a training time of more than 10 years and with more than 3 years of experience in the psychiatric unit were identified. However, their education and experience have little to do with mental health. Young, single, first-time, long-term male patients being hospitalized for high-risk of suicide, followed by manifestations of mental disorders related to alcohol and other drug use. Conclusion: The General Hospital's psychiatric unit provides access to nursing care and other health professionals for comprehensive attention to the needs of people with mental disorders. However, the nursing staff needs more training to deal with mental health in such units. The health professionals face a small number of specialization courses in some regions of Brazil.

Descriptors: Mental Health; Nursing; Hospitals General; Inpatient Care Units.

\section{RESUMEN}

Objetivo: caracterizar la unidad psiquiátrica del Hospital General en términos de estructura, organización del trabajo, perfil profesional de la enfermería y tipo de paciente recibido. Método: Se trata de un estudio de caso de observación directa, enfoque cualitativo y cuantitativo, con la participación de diez profesionales de enfermería. Resultados: Se observó una unidad con una estructura física moderna basada en la intervención multidisciplinar. Jóvenes profesionales de enfermería, mujeres, con más de 10 años de formación y más de 3 años de experiencia en la unidad psiquiátrica, pero cuya formación y experiencia no se refieren a la salud mental. Pacientes varones, jóvenes, solteros, en su primera hospitalización, con una larga estancia, siendo hospitalizados por alto riesgo de suicidio, seguidos de manifestaciones de trastornos mentales relacionados con el consumo de alcohol y otras drogas. Conclusión: La unidad psiquiátrica del hospital general proporciona acceso a la atención de enfermería y a otros profesionales para la atención integral de las necesidades de las personas con trastornos mentales. Sin embargo, es necesario seguir capacitando al personal de enfermería para que se ocupe de la salud mental en estas unidades, que se enfrentan a una oferta reducida de cursos de especialización en algunas regiones de Brasil.

Descriptores: Salud Mental; Enfermería; Hospitales Generales; Unidades de Internación.

'Doutora em Ciências pela Escola de Enfermagem de Ribeirão Preto, Universidade de São Paulo. Docente do Instituto Multiprofissional em Saúde da Universidade Federal da Bahia. ${ }^{2}$ Doutora em Enfermagem Psiquiátrica pela Universidade de São Paulo. Docente da Escola de Enfermagem de Ribeirão Preto, Universidade de São Paulo.

Como citar este artigo:

Alves TC, Luis MAV. Unidade psiquiátrica em hospital geral: características de estrutura e organização. Revista de Enfermagem do Centro Oeste Mineiro. 2020;10:e3470. [Access__]; Available in:__. DOI: http://dx.doi.org/10.19175/recom.v10i0.3470 
Apesar do surgimento de Unidades Psiquiátricas (UP), em diferentes pontos do mundo, houve uma convergência quanto à intencionalidade da organização desses serviços de psiquiatria, no Hospital Geral, que esteve baseada nas críticas de estigma e segregação ao modelo de atendimento que os hospitais psiquiátricos propiciavam aos indivíduos internados, tendo como referência de transformação a experiência de pequenas enfermarias psiquiátricas, em hospitais militares gerais. Nesse contexto, surgiram ações que questionavam a redução das internações em hospitais psiquiátricos, assim como mobilizavam intervenções dos transtornos mentais, nos serviços comunitários de saúde mental, o que incluía a ampliação das Unidades Psiquiátricas em Hospitais Gerais (UPHG) ${ }^{(1)}$.

Houve um crescimento do número de UPHG, sobretudo, na América do Norte, provenientes de fatores como: as críticas ao modelo tradicional de cuidado em psiquiatria, promotor da anomia, da segregação e isolamento; a adoção de uma política de bemestar social, que culminou com a construção de uma rede de proteção e inclusão social, principalmente em países dessa região; a necessidade de integração de estruturas extramuros como necessárias ao cuidado; o desenvolvimento de abordagens socioterapêuticas e psicossociais com vistas à ressocialização e ao reconhecimento da necessidade de incorporar o manejo em psiquiatria na formação profissional, com destaque para a construção de UPHG de ensino ${ }^{(2)}$.

As primeiras UPHG surgiram, no Brasil, na década de 50, em 1954, no Estado da Bahia, com seis leitos destinados às mulheres e um ambulatório de psiquiatria. Inicia-se a expansão de mais UP pelo país, com a implantação, também, nos Estados de São Paulo e Pernambuco ${ }^{(2-3)}$.

As UPs surgem como serviços de saúde mental, situados em uma estrutura hospitalar como uma alternativa aos hospitais psiquiátricos, para o tratamento de pessoas com transtorno mental, álcool e outras drogas, em quadros agudos, tendo ações de superação e crítica ao modelo asilar e segregador, implantadas como propostas assistenciais alternativas às internações prolongadas dos hospitais psiquiátricos tradicionais $^{(3)}$.

Baseado em dados fornecidos, por mais de cento e setenta países ao Atlas da Saúde, houve globalmente uma diminuição de 5\% no número de hospitais psiquiátricos, entre 2011 e 2014, e um aumento em até oito vezes, em algumas regiões do mundo, no que corresponde ao número de leitos psiquiátricos em Hospitais Gerais ${ }^{(4)}$. No Brasil, o Ministério da Saúde aponta um aumento no número de leitos de psiquiatria nos hospitais gerais, com valores que vão de 2.568 leitos em 2005 para 4.620 leitos em $2014^{(5)}$.

Paralelo à ampliação das UPHG, verificaramse mudanças no cuidado em saúde mental no nível hospitalar e, também, na forma de atenção da equipe de enfermagem, nesse contexto, baseado em discussões da necessidade de alteração da lógica manicomial que não individualizava o sujeito, utilizando abordagens com foco no sintoma, no diagnóstico e na fragmentação do paciente ${ }^{(6)}$. Essas provocações culminaram em espaços onde se buscavam a integração do cuidado, que dispensavam atenção aos pacientes psiquiátricos de forma integral e inserida às demais especialidades médicas, com a realização de cuidados que aproximavam a realidade vivida pelo paciente, ao olhar individualizado dos profissionais de saúde, que se baseavam no planejamento das ações de saúde mental, desde a internação até o pós-alta nas relações intersetoriais que incluíam saúde, assistência social, justiça e educação( ${ }^{(7)}$.

Para isso, o cuidado qualificado, deve estar atrelado à capacidade de ser flexível e utilizar diversas abordagens terapêuticas direcionadas à singularidade dos casos e atentas à imprevisibilidade das exposições ${ }^{(8)}$. Esse olhar diferenciado para a loucura, com foco no processo terapêutico do cuidar em saúde, propõe lidar com o ser humano em sua integralidade, por observar o sujeito em seu contexto social na sua totalidade, estando o paciente inserido em qualquer um dos pontos de atenção em saúde mental ${ }^{(9)}$, o que inclui as UPHG.

Nesse contexto, a unidade psiquiátrica em hospital geral, além de ser um equipamento proposto pela rede de cuidado em saúde mental é apontado por estudo ${ }^{(10)}$ como viável e que leva à melhoria do quadro psiquiátrico, contudo sugere conhecer mais detalhadamente a realidade dos serviços de atenção à saúde mental e dos profissionais atuantes nessa área. Assim, surgiu a indagação: como se estrutura uma unidade de psiquiatria no hospital geral, tendo como foco de observação a enfermagem e os pacientes por ela atendidos? 
A presente pesquisa relata uma experiência de trabalho numa unidade de psiquiatria em um Hospital Geral Universitário que passou pelo processo de adequação, avaliação e habilitação recente (2015), pelo Ministério da Saúde, que tiveram como base, portarias específicas de rede de cuidado psicossocial e de serviços hospitalares de referência em saúde mental. Dessa forma, neste estudo, objetivou-se caracterizar a unidade de psiquiatria do Hospital Geral, no tocante à estrutura, forma como organiza o trabalho, ao perfil profissional da enfermagem e ao tipo de paciente que recebe.

\section{METODOLOGIA}

Trata-se de um estudo de caso ${ }^{(11)}$, pautado em uma investigação empírica, atual, dentro de um contexto da vida real, sem definição clara entre fenômeno e contexto estudado, realizado numa abordagem qualitativa e quantitativa ${ }^{(12)}$, que buscou organizar dados sociais, mantendo o caráter unitário do objeto social.

A pesquisa foi realizada em um hospital universitário, do Nordeste do Brasil, tendo sido escolhido por ser um dos últimos hospitais gerais a ter os leitos habilitados para internação em psiquiatria do país. Possui uma equipe multiprofissional, formada por profissionais médicos, enfermeiros, terapeuta ocupacional, psicólogos, profissional de educação física, assistente social, nutricionista e farmacêutica que se dividem no atendimento à psiquiatria, outras especialidades e também à unidade ambulatorial.

Fizeram parte do estudo dez profissionais de enfermagem que compõem a equipe, sendo cinco enfermeiros e cinco técnicos de enfermagem, de um total de 50 profissionais de enfermagem que atuam na UP. Foram sorteados aleatoriamente, de acordo aos seguintes critérios de inclusão/exclusão: ser da equipe de enfermagem; prestar cuidados de enfermagem às pessoas com transtorno mental, álcool e outras drogas, internados e ser sorteado para participar do estudo.

Estabelecer tais critérios foi necessário, em razão de que, nem todos os profissionais de enfermagem atuam, regularmente, prestando cuidados às pessoas com transtornos mentais, nem tampouco havia disponibilidade de horário para a participação nos procedimentos da pesquisa, assim, a escala de trabalho foi um norteador da seleção dos sujeitos para o sorteio, buscando atingir a mesma representatividade de enfermeiros e técnicos.
Utilizou-se, na coleta de dados, a observação direta não participante, com verificação de dados em documentos institucionais ${ }^{(11)}$ relativos às características sociodemográficas e de internação de internados, no período de 2017, perfazendo um número de 79 pacientes internados na UPHG. Assim como se utilizou também questionário autoaplicável, para levantamento de dados demográficos e educacionais dos profissionais.

O material proveniente dos documentos do hospital e a observação subsidiaram a caracterização da estrutura da UP e dos pacientes atendidos. Por meio de questionários autoaplicados, foi possível traçar o perfil dos profissionais de enfermagem participantes do estudo.

Os dados processados, alusivos aos documentos e questionários, foram repassados para construção dos bancos de dados sob a forma de planilhas do aplicativo Microsoft Excel, que foram validadas mediante dupla entrada de dados. Para cruzamento desses dados e distribuição em tabelas de frequência foi empregado o programa SPSS (Statistical Package for the Social, versão 21 para Windows).

O projeto de pesquisa foi submetido e aprovado pelo Comitê de Ética em Pesquisa, da Escola de Enfermagem de Ribeirão Preto, Universidade de São Paulo, conforme resolução no 196/96 do Conselho Nacional de Saúde, e sua atualização, por meio da Resolução no 466 12012, conforme Protocolo CAAE: 79851417.0.0000.5393.

\section{RESULTADOS E DISCUSSÃO}

A apresentação está dividida em uma descrição dos aspectos estruturais do serviço, como: estrutura física, organização do trabalho, recursos humanos e, ainda, o tipo de clientes que são atendidos no serviço.

O hospital estudado, nasceu em 1909, passando por diversas modificações até o ano de 1955, quando se torna um hospital-escola, modificando-se até a obtenção dos moldes atuais. Apesar de existir há muito tempo, verificou-se, com as adequações, ampliações e reformas, uma estrutura que mescla espaços antigos e modernos, com instalações novas e conservadas, mantendo, conforme divisão de setores, um padrão estrutural, com um setor de internação, Edifício Central de Internação (ECI), com 242 leitos de internação, destes 19 leitos de Unidade de Terapia Intensiva. O ECl e a UPHG 
passam a ser uma extensão da estrutura original, seguindo um padrão estrutural para todas as especialidades médicas existentes (psiquiatria, neurologia, neurocirurgia, dentre outras).

Fundada, em 2011, e habilitada pelo Ministério da Saúde, em 2015, a UPHG trouxe como objetivos atender à crise em saúde mental de forma integrada e interdisciplinar; priorizar os casos que necessitem de mais ampla atenção clínica; reinserir o paciente na rede de atenção à saúde mental, com reestruturação de laços familiares e sociais; promover autonomia para dar continuidade ao seu tratamento e criar um espaço apropriado para ensino, extensão e pesquisa, priorizando adequação da assistência para construção e divulgação de conhecimento na área de saúde mental.

O cuidado terapêutico é proposto a partir de recursos técnicos - evolução clínica: psicofarmacologia, suporte clínico, interconsultas de outras especialidades, exames laboratoriais e de imagem, atendimento à crise - protocolo; grupos operativos com pacientes e familiares; intervenções terapêuticas individuais e em grupo e com familiares; reuniões técnicas para a construção e reavaliação dos projetos terapêuticos; supervisão clínico institucional; oficinas terapêuticas; espaços de lazer e cultura, e, por fim, matriciamento com a rede - comunicação com equipes de saúde de outras instituições para continuidade e integralidade da assistência ao paciente.

Para alcançar os objetivos e realizar as intervenções propostas, conta com uma equipe multiprofissional, responsável pela assistência à saúde mental: psiquiatras, enfermeiros, assistente social, psicólogo, farmacêutico, terapeuta ocupacional, que conta com uma assessoria técnica, um supervisor de equipe e, também, com a residência médica em Psiquiatria, serviço de internato em Psiquiatria, estágios em Psicologia, Assistência Social, Terapia Ocupacional, Enfermagem e, posteriormente, as residências multiprofissionais.

A UPHG tem como porta de entrada as unidades setoriais, que são Centro de Atenção Psicossocial, Unidade de Pronto Atendimento, Pronto Socorro, Hospital Especializado, Ambulatório do próprio Hospital Geral que, em contato com o Núcleo Interno de Regulação do Hospital, informa-se acerca da disponibilidade de leitos e programação de alta. Na admissão, é realizado o atendimento/entrevista, por meio do agendamento com médico psiquiatra assistente e um técnico da equipe multiprofissional.

Participam do programa pessoas com transtornos mentais e seu(s) familiar(es) ou responsável(eis). São avaliados os critérios que viabilizem a internação do paciente, de forma a evitar inadequações na admissão. Após esse contato, é planejado o Projeto Terapêutico (PT) inicial com condutas clínico-psiquiátricas e planejamento de atividades terapêuticas e intervenções familiares.

São prioridades para a admissão de pacientes na UP pessoas com transtorno mental da rede que não encontram assistência adequada às suas necessidades, sendo o hospital um serviço complementar aos demais serviços existentes. Observa-se para admissão: possuir comorbidades clínicas significativas: incluindo situações de risco relacionadas à desintoxicação por uso de álcool ou outras substâncias psicoativas, tentativa de suicídio e outros prejuízos clínicos relacionados a transtornos psiquiátricos, em geral; ser idoso, estar exposto a fatores de risco e vulnerabilidade familiar e/ou social e/ou individual; adolescentes em situação de risco: incluindo desintoxicação por uso de álcool ou outras substâncias psicoativas, depressão grave, primeira crise psicótica, transtorno alimentar grave.

A evolução da equipe multiprofissional e as prescrições médicas e de enfermagem são realizadas por meio eletrônico, mediante sistema informatizado; contém evolução médica diária com prescrição, solicitação de exames complementares (laboratoriais e de imagem), solicitação de interconsultas de outras clínicas, solicitação de procedimentos especializados; a evolução da enfermagem, realizada diariamente como condução de rotina da equipe de enfermagem.

O PT iniciado desde a admissão é utilizado de forma interdisciplinar e conta a história do paciente, exames físico e psíquico iniciais, dados sobre família e vínculos sociais. Diariamente, há o registro da evolução tanto das atividades e terapias desenvolvidas pelas pessoas internadas, quanto sua evolução clínica e informações relevantes para reavaliação de seu PT singular. Semanalmente, em dia fixo, a equipe da enfermaria se reúne para reavaliação do PT de cada paciente.

O planejamento de alta se dá de acordo com o PT singular e coletivo e se baseia na evolução e história de cada paciente, após avaliar as necessidades futuras de acompanhamento clínico, 
psíquico e social, com aproximação e comunicação aos demais serviços da rede de cuidado em saúde mental, para dar continuidade ao tratamento. Há preocupação em acompanhar a inserção do paciente nos demais serviços da rede, compartilhando responsabilidades.

$\mathrm{Na}$ alta, agendam-se encontros com pacientes, familiares e membros dos demais serviços que os acolherão, para haver troca de informações sobre o seguimento e cuidado no tratamento. Com isso, busca-se garantir a integração dos serviços e a inserção do paciente na rede, como forma de evitar novas internações.

O serviço prevê agenda de formação com periodicidade semanal com focos diversos como a supervisão clínico institucional da equipe, espaço de reunião da equipe multiprofissional e realizada por profissional supervisor. Vislumbra trabalhar dificuldades institucionais, a partir de capacitação da equipe para lidar com problemas próprios da rotina. Oferta de seminários e oficinas técnicas, a cada dois anos, com discussões sobre a dinâmica do serviço e temas específicos do campo teóricoclínico. Observa-se uma abordagem centrada na figura do médico, não sendo visto, nesses momentos, o protagonismo da atuação da equipe de enfermagem, que fica na posição de espectadora das propostas trazidas pelos profissionais da medicina e também da psicologia.

Dessa forma, a instituição hospitalar é tida como o ambiente que congrega todo o processo de cuidado, com sua organização e características, tornando-se o local que permite que os profissionais atuem conforme as suas potencialidades, evidenciando a influência da estrutura e do processo organizacional para a melhoria do cuidado prestado ${ }^{(13)}$. É evidente que essa mesma organização e características podem tolher o potencial dos trabalhadores e dificultar a realização do cuidado.

A UP encontra-se instalada no $\mathrm{ECl}, 2$ 응 subsolo. A estrutura física se divide em hall de entrada, contendo elevadores, saída de emergência, banheiros sociais e sala de suporte à nutrição; recepção com sala de arquivamento; salas de aula; salas de conforto para os profissionais; copa; sala de procedimentos; sala de prescrição; posto de enfermagem; sala de convivência, banheiros sociais; sala de depósito de materiais; expurgo; enfermarias, sendo uma de isolamento. Cada uma delas possui três leitos, banheiro, lavabo e seus utensílios de acolhimento e acomodação, como leitos, poltronas para acompanhantes, mesa de suporte para alimentação, armários para guardar objetos pessoais. As enfermarias, feminina e masculina, são direcionadas aos leitos de psiquiatria, encontram-se próximas ao posto de enfermagem como forma de melhor observação e acompanhamento dos casos.

Verificou-se que a organização do mobiliário gera redução da área de circulação de pessoas, principalmente, no que diz respeito à sala de prescrição, espaço de circulação de profissionais, estudantes e professores, sendo fator dificultador no processo de trabalho, o que coaduna com estudo ${ }^{(13)}$ realizado em alguns Estados dos Estados Unidos e no Distrito de Colúmbia com enfermeiros que atuam em hospitais universitários que constataram fatores negativos no ambiente de trabalho e influenciam no compromisso organizacional, impactando no processo de trabalho.

Quanto à ventilação e à iluminação, a UP está localizada no subsolo, sendo assim, um local climatizado, que mantém a iluminação artificial, isento da entrada da luz natural. Resultado semelhante foi encontrado em estudo ${ }^{(14)}$ que, ao avaliar processo de trabalho da enfermagem em saúde mental, no que se refere à estrutura, trouxe à inadequação quanto a luminosidade como um dos fatores referentes à inadequação na estrutura e gerador de estresse no trabalho.

Um ambiente com pouca luminosidade torna-se um espaço sombrio que impacta no estado de humor das pessoas, o que influencia, diretamente, em seu bem-estar. A diminuição da temperatura e a escassez da exposição à luz natural faz com que as pessoas predispostas ao adoecimento mental vivam um tipo de depressão, que é acompanhada de alterações como sono e apetite exacerbado, além de comportamento antissocial ${ }^{(15)}$.

Para a organização dos documentos do hospital em estudo, se utiliza o sistema interno informatizado, tendo a tecnologia, favorecendo o processo de trabalho da equipe multiprofissional. Os dados dos pacientes internados são registrados em prontuários eletrônicos, que possuem dados da admissão à alta hospitalar. Nesses documentos constam: ficha de admissão, prescrição médica e de enfermagem, plano terapêutico singular, evolução da equipe multiprofissional - médica, enfermagem, psicologia, nutrição, terapia ocupacional, serviço social e farmacêutica.

Caracterização sociodemográfica e educacional dos profissionais de enfermagem 
Quanto ao perfil dos dez profissionais de enfermagem que atuam na UPHG, participantes do estudo, verificou-se uma média de idade entre os profissionais de 34,1 anos (Desvio Padrão $\pm 7,4$ ), sexo feminino $(80,0 \%)$. Quanto aos dados referentes à formação profissional, obteve-se número igual quanto à categoria profissional de enfermagem em nível superior $(50,0 \%)$ e nível médio $(50,0 \%)$, com tempo de formação maior ou igual a 10 anos (60,0\%), mais de 3 anos de atuação na UP $(80,0 \%)$, regime de trabalho menor ou igual a $36 \mathrm{~h}(60,0 \%)$ e apenas um vínculo profissional $(60,0 \%)$.

No quesito qualificação profissional, além da formação de base, observou-se que a realização de especialização Lato Sensu, ou curso acima de $360 h$, abrangeu a maioria dos profissionais $(80,0 \%)$, alguns realizaram mais de uma especialização ou curso e maior concentração para área hospitalar $(46,1 \%)$. Na realização de especialização Stricto Sensu, houve minoria (20,0\%), que se concentraram na área de saúde coletiva $(100,0 \%)$. Quanto à experiência e formação em saúde mental, a maioria $(70,0 \%)$ relatou experiência prévia com a saúde mental, contudo, apenas durante estágio na formação profissional $(71,4 \%, \mathrm{~N}=7)$ e outros em atuação nos serviços de saúde mental $(28,6 \%, \mathrm{~N}=7)$, em detrimento da minoria $(30,0 \%)$ que nunca teve contato com a saúde mental antes de atuar na UP.

Em relação à participação em evento com a temática saúde mental, poucos $(30,0 \%)$ referem ter participado, alguma vez na vida, porém a maioria $(80,0 \%)$ reforça a busca de informação sobre saúde mental com a própria equipe de enfermagem e troca informações com profissionais de outras categorias $(60,0 \%)$, da mesma forma com outras instituições que lidam também com saúde mental.

Avaliando o perfil dos profissionais de enfermagem, percebe-se que é uma profissão que tem uma força de trabalho jovem, com $45 \%$ da faixa etária que se concentra entre 25 e 35 anos, sendo, predominantemente, do sexo feminino, $85,1 \%$, com tempo de formação acima de dez anos, mantendo, assim, a tradição e cultura da feminilização da saúde, apesar de existir um crescimento de profissionais do sexo masculino, mais presentes na região norte do país ${ }^{(16)}$.

A literatura aponta que a maioria dos profissionais de enfermagem possui uma realidade do multiemprego, mantendo mais de um vínculo e uma maior exposição a longas horas em jornadas múltiplas de trabalho, impactando, diretamente, em sua saúde. Contudo, esses achados diferem dos dados encontrados neste estudo, pois verificou-se a mudança do padrão organizacional da vida; aumento da qualidade, a partir da redução da sobrecarga do trabalho, vista na redução da carga horária e na diminuição dos vínculos empregatícios ${ }^{(17)}$.

Quanto à formação dos profissionais de enfermagem, estudo sobre formação em enfermagem, no Brasil, apresenta que $63,7 \%$ dos profissionais, impulsionados pela expansão do acesso à educação superior, escolhem ingressar na área, principalmente técnicos de enfermagem. Observa-se a busca de formação, por parte dos profissionais, por possuírem escolaridade superior à exigida para atuar nos seus respectivos postos de trabalho ${ }^{(17)}$. Achado que corrobora com o estudo atual, com ênfase no cuidado em UPHG, chama à atenção a falta de qualificação profissional para o cuidado na referida unidade, que gera distorção entre teoria e prática, por existir privação de conhecimentos dessa área, ao ser comparada a outras $^{(18)}$.

O estudo revela a necessidade de estratégia, sugerindo-se a educação permanente para a capacitação dos profissionais, motivandoos para o trabalho interdisciplinar, por meio da própria prática ${ }^{(19)}$. Fatores como estigma e preconceito à formação em psiquiatria ou saúde mental e dificuldade na oferta de cursos de especialização em algumas regiões do Brasil distanciam profissionais de saúde da formação nessa área.

Nesse contraponto, estudo aponta pouco treinamento dos profissionais para atuarem nos serviços de saúde mental; a falta de preparação, ainda durante a formação; a pouca importância dos gestores das instituições em capacitar e aperfeiçoar os saberes e práticas de seus profissionais nessa área, o que gera um comprometimento na qualificação profissional e do cuidado ${ }^{(18)}$.

\section{Características sociodemográficas dos pacientes internados}

No processo de caracterização do perfil dos pacientes internados durante o período de um ano, observou-se uma média de idade de 37,4 anos (Desvio Padrão $\pm 17,7$ ), sexo masculino $(55,7 \%)$, solteiro $(64,6 \%)$, ter concluído o ensino médio $(56,7 \%)$, possuir profissão $(67,1 \%)$. Dentre as formas de ingresso na UP, a regulação interna, realizada pela equipe médica do próprio UPHG, 
obteve o maior número de registros $(86,1 \%)$, em detrimento à regulação externa.

Quanto à frequência de internações do paciente na unidade hospitalar, observaram-se casos de primeira internação em UPHG $(84,8 \%)$ e tempo de permanência na unidade, média de 22,1 dias (DP 16,8 dias e IC 95\% 18,3-25,9), com a maior parte dos pacientes internados, por um tempo de permanência classificada como longa (63,3\%), em detrimento da média (22,8\%) e curta (12,9\%) permanência.

Em relação ao tempo de permanência, foi realizada classificação, conforme as normas estabelecidas na Portaria no 148 de 31 de janeiro de 2012, que faz a divisão periódica - curta de 0 a 7 dias, média de 8 a 15 dias e longa acima de 15 dias - como base de cálculo ligado ao custeio anual dos leitos de atenção a pessoas com sofrimento ou transtorno mental e com necessidades de saúde decorrentes do uso de álcool, crack e outras drogas no Hospital Geral(20).

Quanto aos motivos que levaram à internação na UPHG, observou-se que as causas relacionavam-se ao tratamento clínico em saúde mental em situação de risco elevado de suicídio $(39,2 \%)$, seguidos de manifestações dos transtornos mentais relacionadas ao uso de álcool e outras drogas $(27,8 \%)$ e para conter comportamentos desorganizados e/ou disruptivos $(25,3 \%)$, sendo as demais causas relacionadas a tratamentos específicos de intervenções clínicas odontológicas (2,5\%), desintoxicação (1,3\%), urgência clínica médica (1,3\%), urgência pediátrica $(1,3 \%)$ e intervenção cirúrgica de fístula $(1,3 \%)$.

Quanto ao perfil sociodemográfico dos pacientes internados, observaram-se achados semelhantes ao estudo atual no Brasil e no Exterior. Relativos à faixa etária, estudos realizados, no Paraná, com pacientes internados em UPHG ${ }^{(10)}$ e em hospital-escola, com uma amostra de 489 pacientes, na Índia ${ }^{(21)}$, apontaram o predomínio de jovens e adultos jovens, entre 20 e 49 anos. Fase que impacta a realização de etapas relevantes da vida das pessoas, no que diz respeito: à formação profissional, atividades laborais, sedimentação da família, a partir da criação dos filhos, e tudo isso, ao ser afetado por um transtorno mental, influencia negativamente, em decorrência da cronicidade do adoecimento.

Quanto ao sexo, há concordância com estudo realizado em Guarapuava, Paraná, em que $65 \%$ eram homens ${ }^{(10)}$. Quanto ao estado civil, estudo realizado em Porto Alegre, Rio Grande do Sul (RS), com 96 pacientes internados em hospital geral, apontou a predominância da população solteira, sendo $51 \%^{(22)}$.

No que diz respeito à frequência de internações, estudo realizado no Paraná(10), ratificou o achado desta pesquisa, trazendo, respectivamente, que $88,3 \%$ dos pacientes internados na UP estavam em sua primeira internação.

Sobre o tempo de permanência dos internados em UPHG, os estudos apresentaram uma taxa de permanência longa, como o realizado na UPHG de Ribeirão Preto, São Paulo, com tempo médio de internação de 33,5 dias $^{(23)}$. O mesmo foi observado em estudo internacional, como no Canadá, em 57 hospitais gerais tendo uma média de 20,3 dias de internação ${ }^{(24)}$. Porém, não corroboram com o estudo atual os dados de estudo realizado na Índia ${ }^{(21)}$ que mostrou uma taxa média de permanência de $54 \%$ dos pacientes, com média de 13 dias de internação.

Quanto aos motivos de internação, estudo realizado na rede de atenção psicossocial de Porto Alegre, RS, apontou que $47,9 \%$ das internações estavam relacionadas ao risco ou tentativa de suicídio, o que coaduna com os dados deste estudo ${ }^{(22)}$. Contudo, estudo realizado no Paraná traz o uso de álcool e outras drogas como um dos achados de maior prevalência no que se refere aos motivos das internações em UPHG(10), mas verificou-se a presença de ambos os motivos nas internações, como fatores de maior prevalência em UPHG.

\section{CONSIDERAÇÕES FINAIS}

O hospital em estudo, apesar de antigo, investiu em adequações para sediar uma enfermaria psiquiátrica de hospital geral com espaço adequado e nos moldes para formação e pesquisa, embora apresente problemas de iluminação, climatização e ventilação.

No processo organizacional, foi possível identificar a proposta terapêutica de cuidado, com uma equipe de profissionais diversificada, incluindo o enfermeiro, complementada com atividade de apoio matricial dos casos atendidos, dando a essa unidade hospitalar peculiar que a difere da realidade nacional.

Sua equipe de enfermagem apresenta quantitativamente um número apropriado ao trabalho que exercem, mas apesar de apresentarem formação em nível de especialização e inclusive há enfermeiros com mestrado, essa capacitação não está direcionada para a saúde mental. Também não foi verificado 
nenhum tipo de formação, ou treinamento prévio em saúde mental, a existência de qualificação, durante o exercício da profissão. O que não deixa de causar estranheza, pois o investimento feito foi em qualificar-se na área hospitalar e em saúde coletiva, no entanto, para as atividades que desempenham no cotidiano de trabalho, não houve nenhuma iniciativa que revelasse o seu interesse pela área. Isso é preocupante e leva a inferir que, possivelmente, esses profissionais estão encarando o serviço que exercem como uma etapa com vistas a se deslocar para uma outra área da enfermagem. Contudo, tal hipótese somente poderá ser verificada com pesquisas posteriores.

Em relação às características específicas dessa enfermagem, destaca-se que o tempo de trabalho não é colocado como excessivo, posto que trabalhem apenas em uma instituição, o que demonstra refletir condições propícias para uma boa qualidade de vida desses profissionais, considerando apenas o esforço laboral, não sendo possível inferir algo a respeito do trabalho desempenhado fora da unidade de trabalho.

Quanto aos pacientes atendidos, percebemse similaridades com outras unidades dessa natureza, existentes no Brasil e em outros países. Trata-se tanto de casos de situações de emergência quanto tentativa de suicídios, como os transtornos de humos e uso de substâncias psicoativas, portanto, isso indica que os profissionais enfermeiros que venham a atuar ou atuem nessas unidades precisam se capacitar no cuidado peculiar a essas condições mentais específicas.

A unidade psiquiátrica, no hospital geral, possibilita o acesso ao cuidado de enfermagem e dos demais profissionais para uma atenção integral às necessidades das pessoas com transtorno mental. Além disso, ao proporcionar o contato facilitado com outros serviços comunitários, por meio do esforço do matriciamento, favorece a adesão do paciente à continuidade do seu tratamento. Entende-se a necessidade se essa postura de um serviço seja constante em outras enfermarias de hospitais gerais e nos outros serviços comunitários, melhorando a qualidade do cuidado oferecido aos pacientes e facilitaria a sua inserção e a do familiar no tratamento.

Por fim, vale destacar que o estudo ora apresentado expõe limitações, por restringir-se a apenas uma unidade de psiquiatria de hospital geral, sendo necessária uma caracterização de mais unidades para proporcionar maior discussão acerca da qualidade do cuidado em saúde mental no que diz respeito às questões estrutural, processual e organizacional da instituição em foco.

0 estudo trouxe um importante conhecimento do processo estrutural e organizacional da unidade de psiquiatria de hospital geral, com o foco na equipe de enfermagem e vislumbra contribuir na construção de estratégias de trabalho que vise à melhoria da assistência em saúde mental, tanto referente à estrutura quanto à qualificação dos profissionais para o cuidado às pessoas com transtorno mental.

\section{REFERÊNCIAS}

1. Fagundes Júnior HM, Desviat M, Silva PRF. Reforma psiquiátrica no Rio de Janeiro: Situação atual e perspectivas futuras. Ciênc Saúde Coletiva 2016;21(5):1449-60. DOI: 10.1590/141381232015215.00872016

2. Botega NJ. Práticas de psiquiatria no hospital geral: Interconsultas e emergência. 4a ed. Porto Alegre: Artemed; 2017.

3. Fernandes MM, Duarte ML. Atuação de uma enfermeira em uma unidade de internação psiquiátrica de um Hospital Geral: Relato de experiência. Cadernos Brasileiros de Saúde Mental 2014 [citado em 24 jul 2019]; 6(13):148. Available in:

http://incubadora.periodicos.ufsc.br/index.php/c bsm/article/view/2984

4. World Health Organization (WHO). Mental health atlas. Geneva: WHO; 2014.

5. Brasil, Ministério da Saúde. Saúde Mental em Dados 2015 [citado em 24 jul 2019]; 10(12):5-47. Available in: https://www.mhinnovation.net/ sites/default/files/downloads/innovation/reports/R eport_12-edicao-do-Saude-Mental-em-Dados.pdf. 6. Muniz MP, Tavares CMM, Abrahão $A L$, Souza AC. A assistência de enfermagem em tempos de reforma psiquiátrica. Rev Port Enferm Saúde Mental 2015 [citado em 24 jul 2019]; 13:615. Available in: http://www.scielo.mec.pt/scielo.php?script=sci_a rttext\&pid=S1647-21602015000200008\&lng=pt.

7. Silva L, Maftum MA, Kalinke LP, Mathias TAF, Ferreira ACZ, Capistrano FC. Perfil sociodemográfico e clínico dos pacientes em tratamento na unidade psiquiátrica de um hospital geral. Cogitare Enferm. 2015;20(1):112-20. DOI: 10.5380/ce.v20i1.36414

8. Nicacio TR, Toledo VP, Garcia APRF. Da alienação à clínica da enfermagem: Cuidado aos pacientes psiquiátricos com comorbidade. Rev 
Bras Enferm. 2018;71(supl 5):2229-36. DOI: 10.1590/0034-7167-2017-0930

9. Trajano M, Bernardes SM, Zurba MC. O cuidado em saúde mental: Caminhos possíveis na rede de atenção psicossocial. Cadernos Brasileiros de Saúde Mental 2018 [citado em 12 jul 2019]; 10(25):20-37. Available in: http://incubadora.periodicos.ufsc.br/index.php/c bsm/article/view/5001

10. Silva TL, Maftu MA, Kalinke LP, Mathias TAF, Ferreira ACZ, Capistrano FC. Perfil de internações hospitalares em unidade psiquiátrica de um hospital geral. Rev Min Enferm. 2014;18(3):644-65. DOI: 10.5935/14152762.20140047

11. Yin RK. Estudo de caso: Planejamento e métodos. 5a ed. Porto Alegre: Bookman; 2015.

12. Moreira DA. O método fenomenológico na pesquisa. São Paulo: Pioneira; 2002.

13. Brewer CS, Kovner CT, Djuhic M, Fatehi F, Greene W, Chacko TP, et al. Impact of transformational leadership on nurse work outcomes. J Adv Nurs. 2016;72(11):2879-93. DOI: 10.1111/jan.13055

14. Souza IAS, Pereira MO, Oliveira MAF, Pinho PH, Gonçalves RMDA. Processo de trabalho e seu impacto nos profissionais de enfermagem em serviço de saúde mental. Acta Paul Enferm. 2015;28(5):447-53. DOI: 10.1590/19820194201500075

15. Vernilo CZ, Mota MJ. Análise de espaços hospitalares humanizado. Colloquium Socialis 2018;2(3):1-6. DOI: 10.5747/cs.2018.v02.n3.s040

16. Vargas D, Soares J, Ponce TD, Oliveira BB. Enfermeiros de serviços de urgência e emergência psiquiátrica: Análise de perfil profissional e educacional. Cogitare Enferm. 2017;(22)4:1-9. DOI: $10.5380 / c e . v 22$ i4 40704

17. Persegona MFM, Oliveira ES, Pantoja VJC. As características geopolíticas da enfermagem brasileira. Divulg Saúde Debate 2016 [citado em 3 mar 2019]; 56:8-13. Available in: http://cebes.org.br/site/wpcontent/uploads/2016/12/Divulga\%C3\%A7\%C3\% A3o 56 Cofen.pdf

18. Souza MC, Afonso MLM. Saberes e práticas de enfermeiros na saúde mental: Desafios diante da Reforma Psiquiátrica. Gerais, Rev Interinst Psicol. 2015 [citado em 10 mar 2019]; 8(2):332-47. Available in: http://pepsic.bvsalud.org/scielo.php?script=sci a rttext\&pid=S1983-82202015000300004\&lng=pt

19. Andrade ACM, Otani MAP, Higa EFR, Marin MJS, Caputo VG. Cuidado multiprofissional em uma unidade psiquiátrica de um hospital geral. Rev Psicol Divers Saúde 2018;7(1):60-71. DOI: 10.17267/2317-3394rpds.v7i1.1846

20. Brasil, Ministério da Saúde. Portaria no 148, 31 de janeiro de 2012. Define as normas de funcionamento e habilitação do Serviço Hospitalar de Referência para atenção a pessoas com sofrimento ou transtorno mental e com necessidades de saúde decorrentes do uso de álcool, crack e outras drogas, do Componente Hospitalar da Rede de Atenção Psicossocial, e institui incentivos financeiros de investimento e de custeio. Diário Oficial da União 2012.

21. Sharma P, Singh C, Solanki R, Wasim K. A descriptive analysis of patients admitted in short stay ward at psychiatric centre, Jaipur, Rajasthan, India. Int J Res Med Sci. 2017;5(4):1667-72. DOI: 10.18203/2320-6012.ijrms20171284.

22. Zanardo GLP, Silveira LHC, Rocha CMF, Rocha KB. Internações e reinternações psiquiátricas em um hospital geral de Porto Alegre: Características sócio demográficas, clínicas e do uso da Rede de Atenção Psicossocial. Rev Bras Epidemiol. 2017;20(3):460-74. DOI: 10.1590/1980-5497201700030009

23. Machado V, Santos MA. Taxa de permanência hospitalar de pacientes reinternados em hospital psiquiátrico. J Bras Psiquiatr. 2011;60(1):16-22. DOI: 10.1590/S004720852011000100004

24. Chen S, Collins A, Anderson K, Mckenzie K, Kidd S. Patient characteristics, length of stay, and functional improvement for schizophrenia spectrum disorders: A population study of inpatient Care in Ontario 2005 to 2015. Can J Psychiatry 2017;62(12):854-63. DOI: $10.1177 / 0706743716680167$

Nota: Este artigo representa parte da Tese de Doutoramento defendida em dezembro de 2019, Programa de PósGraduação Enfermagem Psiquiátrica, Escola de Enfermagem de Ribeirão Preto, Universidade de São Paulo (EERP/USP). A pesquisa foi financiada pela pesquisadora.

Recebido em: 04/09/2019

Aprovado em: 30/12/2019

Endereço de correspondência:

Tarcísia Castro Alves

Rua Hormindo Barros, no 255 - Bairro Candeias CEP: 450290-094 Vitória da Conquista/BA - Brasil E-mail: tarcisia.castro@ufba.br 